\title{
7-Etil-6-klor-2-metilkromon ve 7-Etil-6-klorflavonun Sentezi ve Teorik Hesaplamaları
}

\author{
Bayhan KARABULUT ${ }^{*}$ \\ Adlyaman Üniversitesi, Eğitim Fakültesi, Matematik ve Fen Bilimleri Eğitimi Bölümü, Adryaman, Türkiye
} (ORCID:0000-0002-6677-5023)

\begin{abstract}
$\ddot{O} z$
$\mathrm{Bu}$ çalışmada, 7-Etil-6-klor-2-metil kromon ve 7-Etil-6-klorflavon bileșikleri sentezlenmiștir ve teorik spektroskopik özellikleri B3LYP/6-31G(d,p) temel seti ile araştırılmıştır. Sentezlenen bileşiklerin ilk olarak DFT yöntemi ve 6-31G(d,p) temel setinden faydalanılarak optimizasyonları yapılmıştır. Bileşikler için ${ }^{1} \mathrm{H}$ NMR kimyasal kayma değerleri hesaplanmıştır ve teorik olarak hesaplanan değerlerin elde edilen deneysel verilerle uyum içinde olduğu görülmüştür. Deneysel ve teorik kimyasal kayma değerleri için korelasyon grafiği ile regrasyon analizleri yapılmıştır. Ayrıca, çalışllan bileşiklerin en yüksek dolu moleküler orbital enerjisi (HOMO) ve en düşük boş moleküler orbital enerjisi (LUMO), bağ uzunlukları, bağ açıları ve Mulliken atomik yük değerleri hesaplanmıştır. HOMO ve LUMO enerji değerlerinden yararlanılarak iyonizasyon potansiyeli, elektron ilgisi, elektronegatiflik, kimyasal sertlik, kimyasal yumuşaklık gibi parametrelerin hesaplamaları yapılmışıtır.
\end{abstract}

Anahtar kelimeler: Kromon, Flavon, Molekül Orbital, HOMO-LUMO, Yoğunluk Fonksiyonel Teorisi

\section{Synthesis and Theoretical Calculations of 7-Ethyl-6-chloro-2- methylchromone and 7-Ethyl-6-chloroflavone}

\begin{abstract}
In this study, 7-Ethyl-6-chlor-2-methyl chromone and 7-Ethyl-6-chlorflavone compounds were synthesized and their theoretical spectroscopic properties were investigated with the basis set of B3LYP/6-31G(d,p). The synthesized compounds were first optimized by using the DFT method and 6-31G(d,p) basis set. ${ }^{1} \mathrm{H}$ NMR chemical shift values were calculated and the theoretically calculated values were found to be consistent with the obtained experimental data. The regression analysis was performed with correlation graph for experimental and theoretical chemical shift values. In addition, the highest occupied molecular orbital energy (HOMO) and lowest unoccupied molecular orbital energy (LUMO), bond lengths, bond angles and Mulliken atomic charge values were calculated. The calculations for parameters such as ionization potential, electron affinity, electronegativity, chemical hardness, chemical softness were performed by using HOMO and LUMO energy values.
\end{abstract}

Keywords: Chromon, Flavon, Molecular Orbital, HOMO-LUMO, Density Functional Theory

\section{Giriş}

Kromon (benzopiran-4-on) yapısı flavonlar, izoflavonlar, flavonoller gibi flavonoidlerin temel halkasını oluşturur. Kromonlar (benzopiran), piran halkasına kaynaşmış benzen halkası içerir ve çeşitli polifenollerin temel yapılarını oluşturan heterosiklik bileşiklerdir. Hem doğal hem de sentetik kromon türevlerinin bazılarının antitümör, anti-vasküler [1], antimikrobiyal [2], antioksidan [3], TNF- $\alpha$ inhibitörü [4], antifungal [5], antikoagülan, antispazmolitik, östrojenik [6], antiviral [7], antihelmintik, antikanser [8], anti-HIV [9], antitüberküler [10], anti-inflamatuar [11], herbisidal, analjezik ve antikonvülzan [12] aktivite gösterdikleri bilinmektedir. Flavonoidler 15 karbon atomu üzerine kurulu (C6-C3-C6), molekül ağırlığı düşük ve bitkilerde bulunan polifenolik bileşiklerin büyük bir sınıfını

"Sorumlu yazar: bayhankarabulut@gmail.com

Geliş Tarihi:22.04.2019, Kabul Tarihi: 01.08.2020 
temsil ederler. Kroman halkası ile birlikte C-2, C-3 yada C-4 konumunda ikinci bir aromatik halkaya sahiplerdir. Küçük moleküllü heterosiklik bileşikler birçok ilacın önemli farmakoforları olmuştur [13]. Örneğin E vitamini antioksidan aktiviteye sahip, doğal olarak meydana gelen ve yapısında kroman halkası bulunan bir bileşiktir [14]. Flavonoid bileşikleri antiviral, antioksidan, antikanser, antiinflamatuar [15], antibiyotik, antineoplastik, prooksidan, anti-hepatotoksik, vitaminler (vasküler koruma) ve anti-ülserojenik [16] gibi çeşitli biyolojik aktiviteler sergilerler. Dolayısıyla flavanoidler farmakolojik olarak umut verici bileşikler oldukları için sentezleri ve yapılarının aydınlatılması organik kimyada yaygın bir uygulama alanı bulmuştur.

\section{Materyal ve Metot}

Çalışmada 4-klor-3-metilfenol (\%99), 4-klor-3-etilfenol (\%97), etil asetoasetat (\%99) ve etil benzoilasetat ( $\geq \% 90)$, Sigma-Aldrich marka kullanıldı. ${ }^{1} \mathrm{H}$ NMR spektrumları sifir noktası referans1 olarak TMS kullanılarak Varian EM 360L NMR spektrometresiyle, IR spektrumları saf örneklerle Perkin Elmer Spectrum 100 FT-IR spektrometresiyle alınd1, erime noktaları da Electrothermal 9100 Erime Noktası Tayin cihazıyla belirlendi.

\subsection{Sentez}

\subsubsection{7-Etil-6-klor-2-metilkromonun Sentezi (Bileşik I)}

7-Etil-6-klor-2-metilkromonun sentezi Şekil 1'de verilmiştir. $50 \mathrm{~mL}$ 'lik bir erlende, 3-etil-4-klorfenolün $(1,56 \mathrm{~g}, 10 \mathrm{mmol})$ etil asetoasetattaki $(1,5 \mathrm{~mL}, 12 \mathrm{mmol})$ çözeltisine, polifosforik asit $(16 \mathrm{~g}, 47 \mathrm{mmol})$ eklendi. Viskoz bir sıvı olan polifosforik asitle diğer maddelerin iyice karışması sağlandı. Reaksiyon karışımı su banyosunda $75-80{ }^{\circ} \mathrm{C}$ 'de 1 saat 1sıtıldı. Reaksiyon tamamlandıktan sonra, soğutulan sarı renkli viskoz karışıma buzlu su katılarak polifosforik asit hidroliz edildi. Elde edilen suda çözünmeyen katı, vakumdan geçirilerek süzüldü ve su ile yıkandı. Açık havada süzgeç kâğıdı üzerinde kurutulan ham ürün etil alkolde kristallendirildi. Verim: $1,36 \mathrm{~g}(\% 61)$. e.n: $96^{\circ} \mathrm{C}$

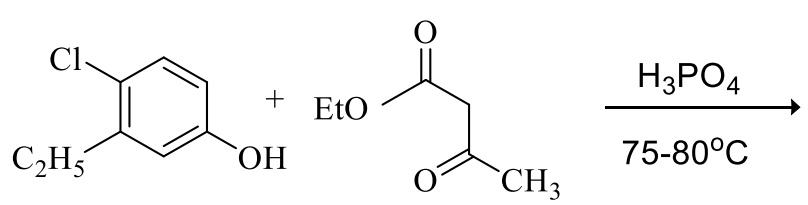

Şekil 1. 7-Etil-6-klor-2-metilkromonun sentezi<smiles>CCc1cc2oc(C)cc(=O)c2cc1Cl</smiles>

(I)

\subsubsection{7-Etil-6-klorflavonun Sentezi (Bileşik II)}

7-Etil-6-klorflavonun sentezi Şekil 2'de verilmiştir.50 mL'lik bir erlende 3-etil-4-klorfenolün (1,56 g, $10 \mathrm{mmol}$ ) etil benzoilasetattaki ( $2 \mathrm{~mL}, 12 \mathrm{mmol})$ çözeltisine, polifosforik asit (16 g, $47 \mathrm{mmol})$ eklendi. Viskoz bir sıvı olan polifosforik asitle diğer maddelerin iyice karışması sağlandı. Reaksiyon karışımı su banyosunda $75-80{ }^{\circ} \mathrm{C}$ 'de 1 saat 1sitıldı. Reaksiyon tamamlandıktan sonra, soğutulan sarı renkli viskoz karışıma buzlu su katılarak polifosforik asit hidroliz edildi. Elde edilen suda çözünmeyen katı, vakumdan geçirilerek süzüldü ve su ile yıkandı. Açık havada süzgeç kâğıdı üzerinde kurutulan ham ürün etil alkolde kristallendirildi. Verim: $0,86 \mathrm{~g}(\% 30)$. e.n: $152{ }^{\circ} \mathrm{C}$<smiles>CCCCCCCCCC(=O)CC(=O)OCC</smiles>

Şekil 2. 7-Etil-6-klorflavonun sentezi<smiles>CCCCCCCCc1cc(=O)c2cc(Cl)c(CC)cc2o1</smiles>

(II) 


\section{Teorik Hesaplamalar}

$\mathrm{Bu}$ çalışmada 7-Etil-6-klor-2-metilkromon ve 7-Etil-6-klorflavon bileşiklerine ait spektroskopik $\left({ }^{1} \mathrm{H}\right.$ NMR, FTIR) ve elektronik özellikler Gaussian G09W paket programında [17], B3LYP (DFT-Density Functional Theory) [18] yöntemi kullanılarak hesaplanmıştır. Her iki moleküle ait teorik hesaplamalar B3LYP/6-31G(d,p) temel seti baz alınarak hesaplanmış ve deneysel verilerle uyumluluğu karşılaştırılmıştır. Teorik hesaplamaların ilk evresinde B3LYP/6-31G(d,p) temel seti ile çalışılan bileşiklere ait optimizasyonlar yapılmıştır. Daha sonra, GIAO [19] metodunda her iki bileşiğin ${ }^{1} \mathrm{H}$ NMR kayma değerleri çözücü olarak kloroform kullanılarak elde edilmiştir. Teorik ${ }^{1} \mathrm{H}$ NMR kimyasal kayma değerleri ile deneysel kimyasal kayma değerleri arasındaki korelasyon incelenmiştir. Ayrıca, bileşiklerin teorik FTIR frekans değerleri HOMO ve LUMO enerjileri, moleküllere ait bağ uzunlukları, bağ açıları ve Mulliken atomik yük değerleri hesaplanmıştır.<smiles>CCc1cc2oc(C)cc(=O)c2cc1Cl</smiles>

(a)

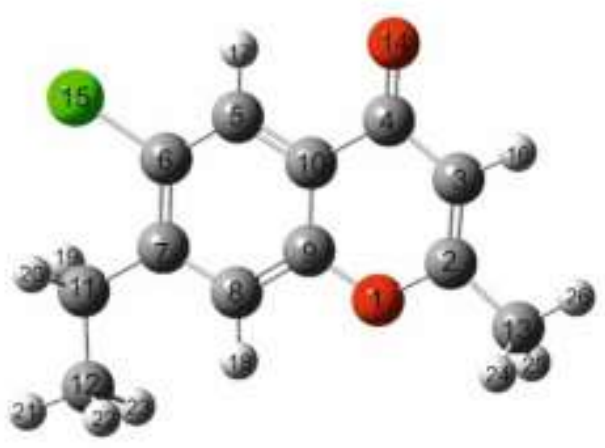

(b)

Şekil 3. (a) 7-Etil-6-klor-2-metilkromon (Bileşik I) için kimyasal yapı ve (b) optimize edilmiş yapı.<smiles>CCc1cc2oc(-c3ccccc3)cc(=O)c2cc1Cl</smiles>

(a)

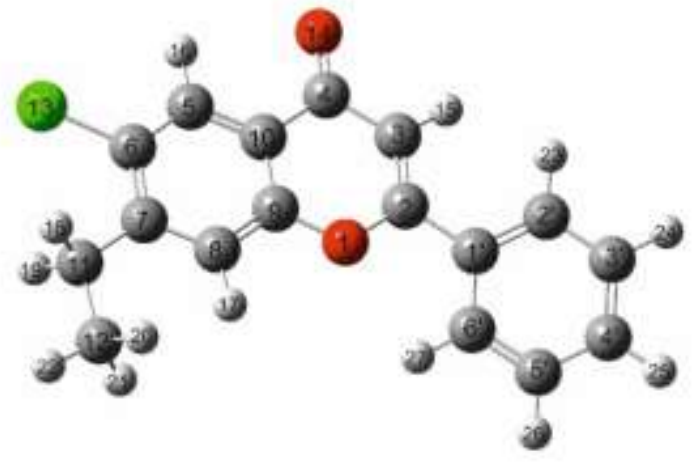

(b)

Şekil 4. (a) 7-Etil-6-klorflavonun (Bileşik II) için kimyasal yapı ve (b) optimize edilmiş yapı.

\section{Bulgular ve Tartışma}

\section{1. ${ }^{1}$ H Nükleer Manyetik Rezonans Spektroskopisi $\left({ }^{1}\right.$ H NMR)}

Bileşik I ve II için deneysel sonuçlarından edilen ve hesaplanan ${ }^{1} \mathrm{H}$ NMR kimyasal kayma değerleri Tablo 1' de verilmiştir. Her iki bileşiğin deneysel ve teorik ${ }^{1} \mathrm{H}$ NMR hesaplamalarında çözücü olarak $\mathrm{CDCl}_{3}$ kullanılmıştır. Bileşik I ve II için deneysel ${ }^{1} \mathrm{H}$ NMR kimyasal kayma değerleri sırasıyla 1,308,10 ppm ve $1,35-8,15$ ppm arasında ortaya çıkarken, teorik kimyasal kayma değerleri sırasıyla 1,28$8,09 \mathrm{ppm}$ ve $1,30-8,10 \mathrm{ppm}$ olarak hesaplanmıştır. Çalış1lan bileşikler için en karakteristik ${ }^{1} \mathrm{H}$ NMR sinyali kromon halkasındaki $-\mathrm{C}=\mathrm{CH}$ protonlarına aittir ve bu protonlar için deneysel ve teorik kimyasal kayma değerleri sırasıyla 6,10 ile 6,03 ppm ve 6,80 ile $6,55 \mathrm{ppm}$ olarak belirlenmiştir. Bu protonların yüksek alanda rezonans olması karbonil grubunda bulunan oksijenin elektronegatifliğinden kaynaklanmaktadır. Bileşik I ve II için deneysel ve teorik ${ }^{1} \mathrm{H}$ NMR kimyasal kayma değerleri arasındaki korelasyon grafikleri Şekil 5'de verilmiştir. Korelasyon katsayısı Bileşik I için 0,997, Bileşik II için ise 
0,993 olarak hesaplanmıştır ki bu değerler bize deneysel verilerin hesaplamalarla büyük bir uyum içinde olduğunu göstermektedir.

Tablo 1. Bileşik I ve II için deneysel ve teorik ${ }^{1} \mathrm{H}$ NMR kimyasal kayma değerleri $\delta$ (ppm)

\begin{tabular}{|c|c|c|c|c|c|c|c|}
\hline \multicolumn{4}{|c|}{ Bileşik I } & \multicolumn{4}{|c|}{ Bileşik II } \\
\hline $\begin{array}{l}\text { Atom } \\
\text { No }\end{array}$ & $\begin{array}{c}\delta \text { (Deneysel) } \\
\left(\mathrm{CDCl}_{3}\right)\end{array}$ & $\begin{array}{c}\delta(\mathrm{B} 3 \mathrm{LYP}) \\
\left(\mathrm{CDCl}_{3}\right)\end{array}$ & $\Delta \delta$ & $\begin{array}{l}\text { Atom } \\
\text { No }\end{array}$ & $\begin{array}{c}\delta \text { Deneysel } \\
\left(\mathrm{CDCl}_{3}\right)\end{array}$ & $\begin{array}{c}\delta \text { B3LYP } \\
\left(\mathrm{CDCl}_{3}\right)\end{array}$ & $\Delta \delta$ \\
\hline H17 & 8,10 & 8,09 & 0,01 & H 16 & 8,15 & 8,10 & $-0,55$ \\
\hline H18 & 7,25 & 7,33 & $-0,08$ & H 23 & 7,55 & 8,10 & 0,05 \\
\hline H16 & 6,10 & 6,03 & 0,07 & H 27 & 7,55 & 7,88 & $-0,33$ \\
\hline H19 & 2,85 & 2,80 & 0,05 & H 26 & 7,55 & 7,61 & $-0,06$ \\
\hline $\mathrm{H} 20$ & 2,85 & 2,80 & 0,05 & H 25 & 7,55 & 7,61 & $-0,06$ \\
\hline $\mathrm{H} 24$ & 2,40 & 2,39 & 0,01 & H 24 & 7,55 & 7,61 & $-0,06$ \\
\hline $\mathrm{H} 25$ & 2,40 & 2,39 & 0,01 & H 17 & 7,90 & 7,41 & 0,49 \\
\hline $\mathrm{H} 26$ & 2,40 & 1,95 & 0,45 & H 15 & 6,80 & 6,55 & 0,25 \\
\hline $\mathrm{H} 21$ & 1,30 & 1,34 & $-0,04$ & H 18 & 2,90 & 2,79 & 0,11 \\
\hline $\mathrm{H} 22$ & 1,30 & 1,28 & 0,02 & H 19 & 2,90 & 2,79 & 0,11 \\
\hline $\mathrm{H} 23$ & 1,30 & 1,28 & 0,02 & H 20 & 1,35 & 1,30 & 0,05 \\
\hline & & & & H 21 & 1,35 & 1,30 & 0,05 \\
\hline & & & & H 22 & 1,35 & 1,30 & 0,05 \\
\hline
\end{tabular}
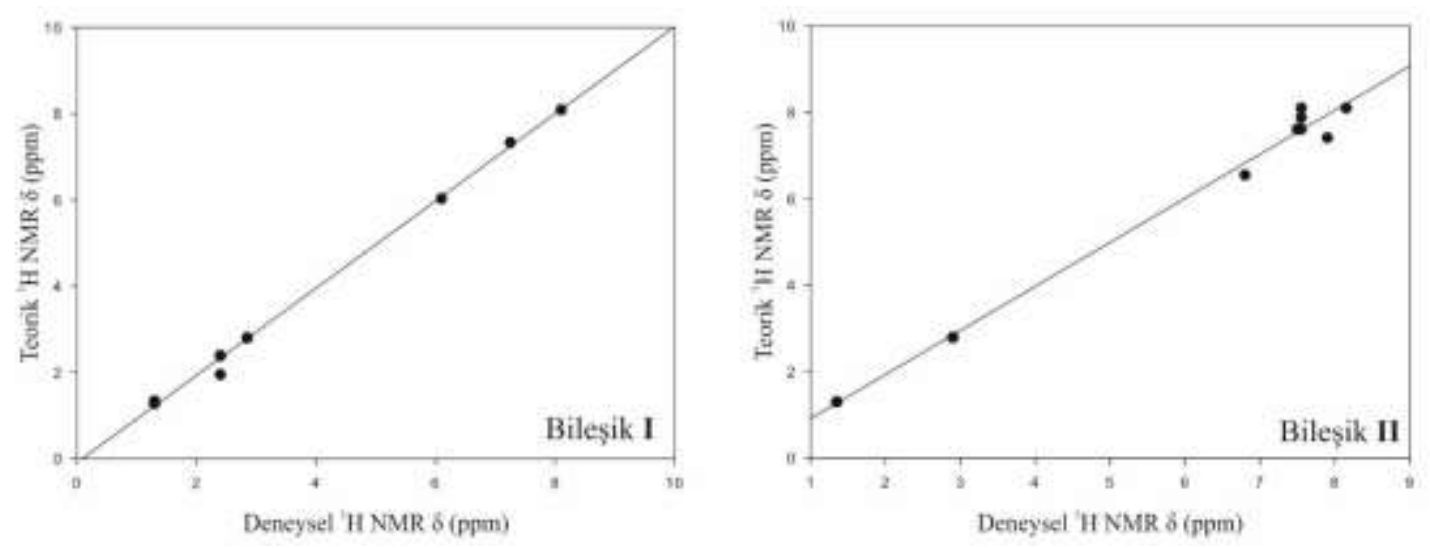

Şekil 5. Bileşik I ve II için deneysel ve teorik ${ }^{1} \mathrm{H}$ NMR kimyasal kayma değerleri arasındaki korelasyon grafikleri.

\subsection{Molekül Geometrisi}

Bileşik I ve II için bağ uzunlukları ve bağ açıları sırasıyla Tablo 2 ve 3 de verilmiştir. Literatürde flavon molekülünün $\mathrm{A}$, B ve $\mathrm{C}$ halkaları için $\mathrm{C}$-C bağ uzunluklarının ortalamaları sırasıyla 1,395, 1,422 ve $1,396 \mathrm{~A}^{\mathrm{o}}, \mathrm{C}=\mathrm{O}$ bağ uzunlukları $1,226 \mathrm{~A}^{\mathrm{o}}$ ve $\mathrm{C}$ halkasına ait $\mathrm{C}-\mathrm{O}$ bağ uzunlukları ise $1,366 \mathrm{~A}^{\mathrm{o}}$ olarak hesaplanmıştır [20]. Çalışmamızda ise A, B ve C halkaları için C-C bağ uzunluklarının ortalaması sırasıyla Bileşik II için 1,395; 1,395; 1,421 $\mathrm{A}^{\mathrm{o}}$ olarak hesaplanmıştır. Bileşik I için C-C bağ uzunlukları ise A halkası için 1,398 ve $\mathrm{C}$ halkası için ise $1,422 \mathrm{~A}^{\circ}$ olarak hesaplanmıştır. $\mathrm{C}=\mathrm{O}$ bağ uzunlukları ise $\mathbf{I}$ ve II bileşiği için sırasıyla 1,229 ve $1,224 \mathrm{~A}^{\mathrm{o}}$ olarak hesaplanmıştır. Her iki bileşik için alifatik C-C tekli bağlarının uzunluklarına ait ortalama değer $1,528 \mathrm{~A}^{\circ}$, C halkasına ait olan $\mathrm{C}-\mathrm{O}$ tekli bağ uzunlukları $\mathbf{I}$. bileşik için 1,$363 ; 1,373$ ve II. bileşik için ise 1,$363 ; 1,370$ olarak hesaplanmış ve hesaplanan bu değerlerin literatürle uyumlu oldukları gözlenmiştir. 
Tablo 2. Bileşik I ve II için bağ uzunlukları

\begin{tabular}{|c|c|c|c|c|c|}
\hline \multicolumn{3}{|c|}{ Bileşik I } & \multicolumn{3}{|c|}{ Bileşik II } \\
\hline \multicolumn{2}{|c|}{ Bağ Uzunluğu $\left(\mathrm{A}^{\circ}\right)$} & B3LYP & \multicolumn{2}{|c|}{ Bağ Uzunluğu $\left(\mathrm{A}^{\mathrm{o}}\right)$} & B3LYP \\
\hline 1 & $\mathrm{O}(1)-\mathrm{C}(2)$ & 1,363 & 1 & $\mathrm{O}(1)-\mathrm{C}(2)$ & 1,363 \\
\hline 2 & $\mathrm{C}(2)-\mathrm{C}(13)$ & 1,496 & 2 & $\mathrm{C}(2)-\mathrm{C}\left(1^{\prime}\right)$ & 1,474 \\
\hline 3 & $\mathrm{C}(2)-\mathrm{C}(3)$ & 1,352 & 3 & $C\left(1^{\prime}\right)-C\left(2^{\prime}\right)$ & 1,402 \\
\hline 4 & $\mathrm{C}(3)-\mathrm{C}(4)$ & 1,460 & 4 & $\mathrm{C}\left(2^{\prime}\right)-\mathrm{C}\left(3^{\prime}\right)$ & 1,389 \\
\hline 5 & $\mathrm{C}(4)-\mathrm{O}(14)$ & 1,229 & 5 & $\mathrm{C}\left(3^{\prime}\right)-\mathrm{C}\left(4^{\prime}\right)$ & 1,394 \\
\hline 6 & $\mathrm{C}(4)-\mathrm{C}(10)$ & 1,482 & 6 & $C\left(4^{\prime}\right)-C\left(5^{\prime}\right)$ & 1,392 \\
\hline 7 & $C(10)-C(5)$ & 1,401 & 7 & $C\left(5^{\prime}\right)-C\left(6^{\prime}\right)$ & 1,390 \\
\hline 8 & $\mathrm{C}(5)-\mathrm{C}(6)$ & 1,382 & 8 & $\mathrm{C}\left(6^{\prime}\right)-\mathrm{C}\left(1^{\prime}\right)$ & 1,402 \\
\hline 9 & $\mathrm{C}(6)-\mathrm{Cl}(15)$ & 1,763 & 9 & $\mathrm{C}(2)-\mathrm{C}(3)$ & 1,355 \\
\hline 10 & $\mathrm{C}(6)-\mathrm{C}(7)$ & 1,416 & 10 & $\mathrm{C}(3)-\mathrm{C}(4)$ & 1,456 \\
\hline 11 & $\mathrm{C}(7)-\mathrm{C}(11)$ & 1,515 & 11 & $\mathrm{C}(4)-\mathrm{O}(14)$ & 1,224 \\
\hline 12 & $\mathrm{C}(11)-\mathrm{C}(12)$ & 1,529 & 12 & $\mathrm{C}(4)-\mathrm{C}(10)$ & 1,480 \\
\hline 13 & $\mathrm{C}(7)-\mathrm{C}(8)$ & 1,393 & 13 & $C(10)-C(5)$ & 1,399 \\
\hline 14 & $\mathrm{C}(8)-\mathrm{C}(9)$ & 1,397 & 14 & $\mathrm{C}(5)-\mathrm{C}(6)$ & 1,378 \\
\hline 15 & $C(9)-C(10)$ & 1,396 & 15 & $\mathrm{C}(6)-\mathrm{Cl}(13)$ & 1,763 \\
\hline 16 & $\mathrm{C}(9)-\mathrm{O}(1)$ & 1,373 & 16 & $\mathrm{C}(6)-\mathrm{C}(7)$ & 1,414 \\
\hline & & & 17 & $\mathrm{C}(7)-\mathrm{C}(11)$ & 1,513 \\
\hline & & & 18 & $\mathrm{C}(11)-\mathrm{C}(12)$ & 1,528 \\
\hline & & & 19 & $\mathrm{C}(7)-\mathrm{C}(8)$ & 1,390 \\
\hline & & & 20 & $\mathrm{C}(8)-\mathrm{C}(9)$ & 1,395 \\
\hline & & & 21 & $\mathrm{C}(9)-\mathrm{C}(10)$ & 1,393 \\
\hline & & & 22 & $\mathrm{C}(9)-\mathrm{O}(1)$ & 1,370 \\
\hline
\end{tabular}

Tablo 3. Bileşik I ve II için bağ açıları

\begin{tabular}{|c|c|c|c|c|c|}
\hline \multicolumn{3}{|c|}{ Bilessik I } & \multicolumn{3}{|c|}{ Bileșik II } \\
\hline \multicolumn{2}{|c|}{ Bağ Açıları $\left(^{\circ}\right)$} & B3LYP & \multicolumn{2}{|c|}{ Bağ Açıları $\left(^{\circ}\right)$} & \multirow{2}{*}{$\begin{array}{r}\text { B3LYP } \\
112,14\end{array}$} \\
\hline 1 & $\mathrm{O}(1)-\mathrm{C}(2)-\mathrm{C}(13)$ & 111,11 & 1 & $\mathrm{O}(1)-\mathrm{C}(2)-\mathrm{C}\left(1^{\prime}\right)$ & \\
\hline 2 & $\mathrm{C}(13)-\mathrm{C}(2)-\mathrm{C}(3)$ & 126,15 & 2 & $\mathrm{C}(2)-\mathrm{C}\left(1^{\prime}\right)-\mathrm{C}\left(2^{\prime}\right)$ & 120,77 \\
\hline 3 & $\mathrm{C}(2)-\mathrm{C}(3)-\mathrm{C}(4)$ & 122,28 & 3 & $\mathrm{C}\left(1^{\prime}\right)-\mathrm{C}\left(2^{\prime}\right)-\mathrm{C}\left(3^{\prime}\right)$ & 120,53 \\
\hline 4 & $\mathrm{C}(3)-\mathrm{C}(4)-\mathrm{O}(14)$ & 123,86 & 4 & $\mathrm{C}\left(2^{\prime}\right)-\mathrm{C}\left(3^{\prime}\right)-\mathrm{C}\left(4^{\prime}\right)$ & 120,25 \\
\hline 5 & $\mathrm{C}(3)-\mathrm{C}(4)-\mathrm{C}(10)$ & 113,39 & 5 & $\mathrm{C}\left(3^{\prime}\right)-\mathrm{C}\left(4^{\prime}\right)-\mathrm{C}\left(5^{\prime}\right)$ & 119,65 \\
\hline 6 & $\mathrm{O}(14)-\mathrm{C}(4)-\mathrm{C}(10)$ & 122,74 & 6 & $\mathrm{C}\left(4^{\prime}\right)-\mathrm{C}\left(5^{\prime}\right)-\mathrm{C}\left(6^{\prime}\right)$ & 120,30 \\
\hline 7 & $\mathrm{C}(4)-\mathrm{C}(10)-\mathrm{C}(5)$ & 121,43 & 7 & $\mathrm{C}\left(5^{\prime}\right)-\mathrm{C}\left(6^{\prime}\right)-\mathrm{C}\left(1^{\prime}\right)$ & 120,46 \\
\hline 8 & $\mathrm{C}(10)-\mathrm{C}(5)-\mathrm{C}(6)$ & 119,98 & 8 & $\mathrm{C}\left(6^{\prime}\right)-\mathrm{C}\left(1^{\prime}\right)-\mathrm{C}(2)$ & 120,43 \\
\hline 9 & $\mathrm{C}(5)-\mathrm{C}(6)-\mathrm{Cl}(15)$ & 118,15 & 9 & $\mathrm{C}\left(1^{\prime}\right)-\mathrm{C}(2)-\mathrm{C}(3)$ & 125,96 \\
\hline 10 & $\mathrm{C}(5)-\mathrm{C}(6)-\mathrm{C}(7)$ & 122,35 & 10 & $\mathrm{C}(2)-\mathrm{C}(3)-\mathrm{C}(4)$ & 122,70 \\
\hline 11 & $\mathrm{Cl}(15)-\mathrm{C}(6)-\mathrm{C}(7)$ & 119,80 & 11 & $\mathrm{C}(3)-\mathrm{C}(4)-\mathrm{O}(14)$ & 123,73 \\
\hline 12 & $\mathrm{C}(6)-\mathrm{C}(7)-\mathrm{C}(11)$ & 120,82 & 12 & $\mathrm{C}(3)-\mathrm{C}(4)-\mathrm{C}(10)$ & 113,44 \\
\hline 13 & $\mathrm{C}(7)-\mathrm{C}(11)-\mathrm{C}(12)$ & 116,22 & 13 & $\mathrm{O}(14)-\mathrm{C}(4)-\mathrm{C}(10)$ & 122,82 \\
\hline 14 & $\mathrm{C}(11)-\mathrm{C}(7)-\mathrm{C}(8)$ & 122,07 & 14 & $\mathrm{C}(4)-\mathrm{C}(10)-\mathrm{C}(5)$ & 121,58 \\
\hline 15 & $\mathrm{C}(7)-\mathrm{C}(8)-\mathrm{C}(9)$ & 120,73 & 15 & $\mathrm{C}(10)-\mathrm{C}(5)-\mathrm{C}(6)$ & 120,00 \\
\hline 16 & $\mathrm{C}(8)-\mathrm{C}(9)-\mathrm{C}(10)$ & 121,60 & 16 & $\mathrm{C}(5)-\mathrm{C}(6)-\mathrm{Cl}(13)$ & 118,14 \\
\hline 17 & $\mathrm{C}(8)-\mathrm{C}(9)-\mathrm{O}(1)$ & 116,59 & 17 & $\mathrm{Cl}(13)-\mathrm{C}(6)-\mathrm{C}(7)$ & 119,49 \\
\hline 18 & $\mathrm{O}(1)-\mathrm{C}(9)-\mathrm{C}(10)$ & 121,79 & 18 & $\mathrm{C}(6)-\mathrm{C}(7)-\mathrm{C}(11)$ & 120,84 \\
\hline & & & 19 & $\mathrm{C}(7)-\mathrm{C}(11)-\mathrm{C}(12)$ & 116,28 \\
\hline & & & 20 & $\mathrm{C}(6)-\mathrm{C}(7)-\mathrm{C}(8)$ & 117,07 \\
\hline & & & 21 & $\mathrm{C}(7)-\mathrm{C}(8)-\mathrm{C}(9)$ & 120,77 \\
\hline & & & 22 & $\mathrm{C}(8)-\mathrm{C}(9)-\mathrm{C}(10)$ & 121,54 \\
\hline & & & 23 & $\mathrm{C}(8)-\mathrm{C}(9)-\mathrm{O}(1)$ & 116,59 \\
\hline & & & 24 & $\mathrm{C}(9)-\mathrm{O}(1)-\mathrm{C}(2)$ & 119,91 \\
\hline
\end{tabular}

\subsection{HOMO-LUMO Enerjileri ve Elektronik Özellikler}

Reaksiyonlara katılan temel orbitaller HOMO ( En Yüksek Dolu Molekül Orbital) ve LUMO (En Düşük Boş Molekül Orbital)'dur. Еномо enerjisi molekülün elektron verme, $E_{\text {LUMo }}$ enerjisi ise molekülün elektron alma yeteneği şeklinde açıklanır. $\mathrm{Bu}$ iki enerji değeri arasındaki fark $\left(\Delta \mathrm{E}=\mathrm{E}_{\text {номо- }} \mathrm{E}_{\mathrm{LUMO}}\right)$ molekülün kimyasal kararlılığının bir göstergesidir ve $\Delta \mathrm{E}$ ne kadar küçükse molekül o kadar yüksek kimyasal reaktiviteye sahiptir [21]. Bileşik I için hesaplanan $\Delta \mathrm{E}=-4,92173$ ve Bileşik II için hesaplanan $\Delta \mathrm{E}=-4,49804 \mathrm{eV}$ 'luk değerler moleküllerin kararlı yapıda olduklarının göstergesidir. HOMO ve LUMO 
enerji değerlerinden yararlanılarak iyonizasyon potansiyeli $\left(\mathrm{I}=-\mathrm{E}_{\text {номо }}\right)$, elektron ilgisi $\left(\mathrm{A}=-\mathrm{E}_{\mathrm{LUMO}}\right)$, elektronegatiflik $(\chi=(I+A) / 2)$, kimyasal sertlik $((\eta=I-A) / 2)$ ve kimyasal yumuşaklık $((S=1 /(2 \eta))$ gibi parametreler hesaplanabilmektedir. Sertlik, bir molekülün polarizasyon kabiliyetinin, yumuşaklık ise elektron alma yeteneğini ölçüsü olarak tanımlanır. Bileşik I ve II için hesaplanan sertlik değerleri sırayla 2,46086, 2,24902 eV, yumuşaklık değerleri ise sırayla 0,20318 ve 0,22232 eV'tur. Elektronegatiflik bağı oluşturan atomların her birinin bağ elektronlarını çekebilme gücü olarak tanımlanır ve kimyasal aktivitenin önemli bir göstergesidir. Bileşik I için hesaplanan elektronegatiflik değeri 3,97354 iken Bileşik II için bu değer 4,40253 eV' tur. Bileșik I ve II için teorik olarak hesaplanan HOMO-LUMO şekilleri ve enerji değerleri Şekil 6' da ve hesaplanan elektronik yapı parametreleri ise Tablo 4' de verilmiştir.

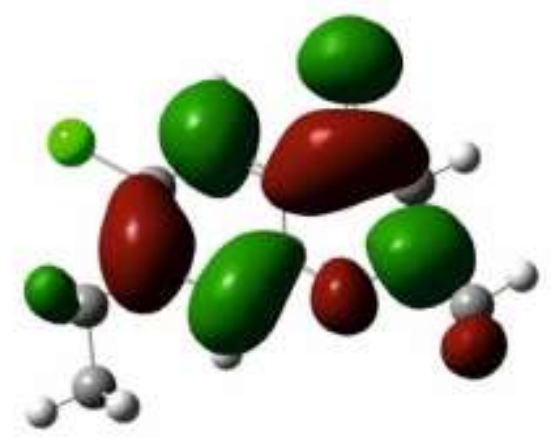

Bileşik I için ELUmo: -1,51268 eV

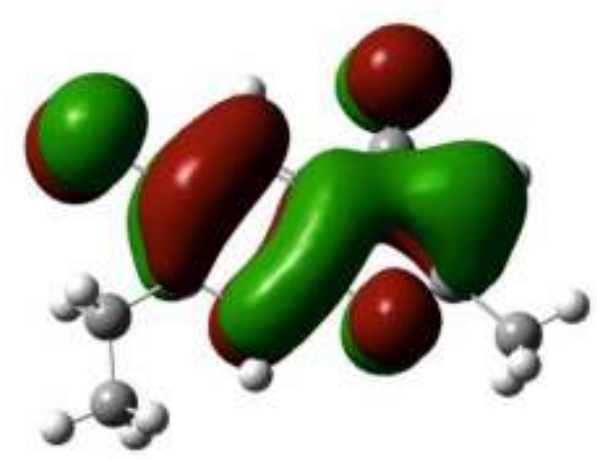

Bileşik I için $\mathrm{E}_{\text {номо: }}-6,43441 \mathrm{eV}$

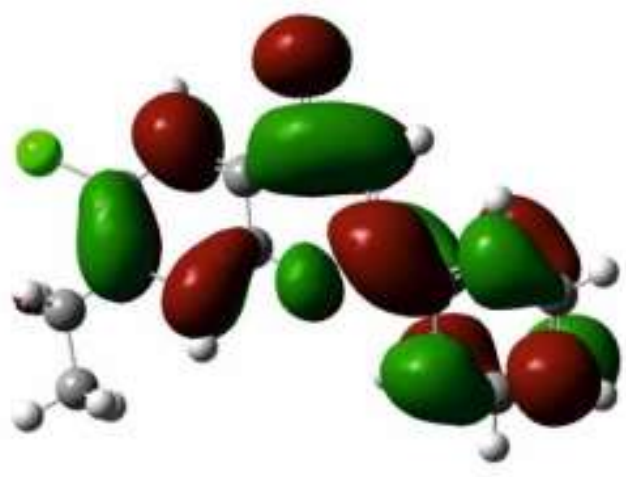

Bileşik II için ELumo: -2,15351 eV

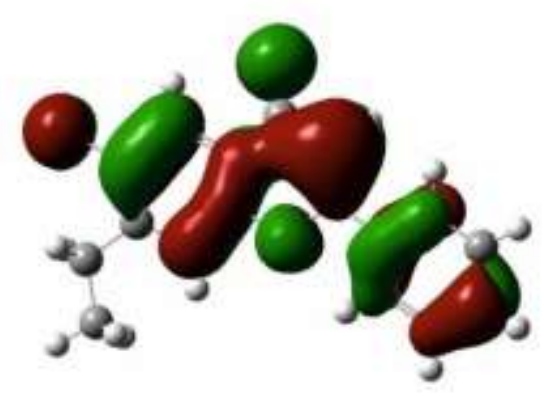

Bileşik II için $\mathrm{E}_{\text {номо: }}-6,65155 \mathrm{eV}$

Şekil 6. Bileşik I ve II için teorik olarak hesaplanan HOMO-LUMO şekilleri ve enerji değerleri

Tablo 4. Bileşik I ve II için hesaplanan elektronik yapı parametreleri

\begin{tabular}{|l|r|r|}
\hline & Bileşik I & Bileşik II \\
\hline Еномо $(\mathrm{eV})$ & $-6,43441$ & $-6,65155$ \\
\hline ELUмо $_{\text {LeV })}$ & $-1,51268$ & $-2,15351$ \\
\hline$\Delta \mathrm{E}=$ Eномо-ELumo $(\mathrm{eV})$ & $-4,92173$ & $-4,49804$ \\
\hline $\mathrm{I}(\mathrm{eV})$ & 6,43441 & 6,65155 \\
\hline $\mathrm{A}(\mathrm{eV})$ & 1,51268 & 2,15351 \\
\hline $\mathrm{X}(\mathrm{eV})$ & 3,97354 & 4,40253 \\
\hline$\eta(\mathrm{eV})$ & 2,46086 & 2,24902 \\
\hline $\mathrm{S}\left(\mathrm{eV}^{-1}\right)$ & 0,20318 & 0,22232 \\
\hline
\end{tabular}




\section{4. İnfrared Spektroskopisi (IR)}

Heteroaromatik bileşikler için IR spektrumunda $3000-3100 \mathrm{~cm}^{-1}$ bölgesi karakteristik C-H gerilme titreşimlerine aittir. Bununla birlikte $\mathrm{C}-\mathrm{H}$ düzlem içi bükülme ve düzlem dışı bükülme titreşimleri sirasiyla $1300-1000 \mathrm{~cm}^{-1}$ ve $1000-750 \mathrm{~cm}^{-1}$ bölgelerinde gözlemlenir. Karbonil grubunun karakteristik titreşim frekansının belirlenmesi amacıyla çok çeşitli bileşikler üzerinde çalışmalar yapılmıştır. $\mathrm{Bu}$ bileşikler için $1850-1550 \mathrm{~cm}^{-1}$ bölgesinde güçlü karbonil titreşimleri gözlenmiştir [22]. Bileşik I ve II için deneysel ve teorik IR titreşim frekans değerleri Tablo 5' de verilmiştir. Flavon molekülü için 1646 $\mathrm{cm}^{-1}$ 'de gözlenen karakteristik pik $\mathrm{C}=\mathrm{O}$ gerilme titreşim frekansına aittir. $\mathrm{C}=\mathrm{C}$ gerilme titreşimleri aromatik bileşikler için genellikle $1430-1650 \mathrm{~cm}^{-1}$ bölgesinde gözlenirken, flavon bileşikleri için $\mathrm{C}=\mathrm{C}$ gerilme titreşimleri tüm halkalarda $1606 \mathrm{~cm}^{-1}$ bölgesinde görülmektedir [20].

Tablo 5. Bileşik I ve II için deneysel ve hesaplanan IR titreşim frekans değerleri

\begin{tabular}{|c|c|c|}
\hline \multicolumn{3}{|c|}{ Bileşik I } \\
\hline \multirow[t]{2}{*}{ Titreşim türleri } & \multicolumn{2}{|c|}{ Titreşim frekansları $\left(\mathrm{cm}^{-1}\right)$} \\
\hline & B3LYP/6-31G(d,p) & Deneysel \\
\hline v CH Arm. & 3105 & 3080 \\
\hline $\mathrm{v} \mathrm{CH}_{3} \mathrm{Arm}$ & 3038 & 2985 \\
\hline$v \mathrm{CH}_{2} \mathrm{CH}_{3} \mathrm{Arm}$. & 3004 & 2960 \\
\hline v $\mathrm{CH}_{3} \mathrm{Arm}$ & 2989 & 2937 \\
\hline $\mathrm{v} \mathrm{CH}_{2} \mathrm{Arm}$. & 2947 & 2917 \\
\hline v $\mathrm{C}=\mathrm{O}$ & 1683 & 1615 \\
\hline$\delta \mathrm{CH}_{2} \mathrm{CH}_{3} \mathrm{Arm}$. & 1463 & - \\
\hline$\delta \mathrm{CH}$ Arm. & 1447 & 1426 \\
\hline$\delta \mathrm{CH}_{3} \mathrm{Arm}$. & 1437 & 1412 \\
\hline$\delta \mathrm{CH}_{2} \mathrm{Arm}$. & 1426 & 1380 \\
\hline$\delta \mathrm{CH}$ Arm. & 1224 & 1233 \\
\hline$\delta \mathrm{CH}$ Arm. & 1211 & 1208 \\
\hline$\delta \mathrm{CH}$ Arm. & 1204 & 1176 \\
\hline \multicolumn{3}{|c|}{ Bileşik II } \\
\hline \multirow[t]{2}{*}{ Titreşim türleri } & \multicolumn{2}{|c|}{ Titreşim frekansları $\left(\mathrm{cm}^{-1}\right)$} \\
\hline & B3LYP/6-31G(d,p) & Deneysel \\
\hline v CH Arm. & 3087 & 3032 \\
\hline v CH Arm. & 3073 & 2982 \\
\hline v CH Arm. & 3045 & 3003 \\
\hline $\mathrm{v} \mathrm{CH}_{3}$ & 2980 & 2907 \\
\hline $\mathrm{v} \mathrm{CH}_{2}$ & 2927 & 2879 \\
\hline $\mathrm{v} \mathrm{C}=\mathrm{O}$ & 1657 & 1632 \\
\hline v CH Arm. & 1580 & 1553 \\
\hline v CH Arm. & 1528 & 1498 \\
\hline$\delta \mathrm{CH}_{3}$ & 1448 & 1435 \\
\hline$\delta \mathrm{CH}$ Arm. & 1422 & 1411 \\
\hline$\delta \mathrm{CH}_{2}$ & 1326 & 1352 \\
\hline$\delta \mathrm{CH}_{2}+\mathrm{CH}_{3}$ & 1253 & 1292 \\
\hline$\delta \mathrm{CH}$ Arm. & 1239 & 1269 \\
\hline
\end{tabular}

৩: gerilme; $\delta$ : düzlem içi eğilme; Arm: Aromatik

\subsection{Mulliken Atomik Yük Dağılımları}

Her iki bileşik için B3LYP/6-31G(d,p) temel seti kullanılarak elde edilen atomik yük değerleri Tablo 6' da verilmiştir. Her iki bileşikte de karbonil grubuna bağlı olan C4 karbon atomunun en yüksek Mulliken atomik yük değerine sahip olması karbona bağlı olan elektronegatif oksijen atomundan kaynaklanmaktadır. 
Tablo 6. Bileşik I ve II Mulliken atomik yük değerleri

\begin{tabular}{|c|c|c|c|}
\hline \multicolumn{2}{|c|}{ Bileşik I } & \multicolumn{2}{|c|}{ Bileşik II } \\
\hline Atom No & B3LYP & Atom No & B3LYP \\
\hline $\mathrm{O} 1$ & $-0,523$ & $\mathrm{O} 1$ & 0,540 \\
\hline $\mathrm{C} 2$ & 0,341 & $\mathrm{C} 2$ & 0,320 \\
\hline $\mathrm{C} 3$ & $-0,192$ & C3 & $-0,193$ \\
\hline $\mathrm{C} 4$ & 0,415 & $\mathrm{C} 4$ & 0,413 \\
\hline C5 & $-0,100$ & C5 & $-0,100$ \\
\hline C6 & $-0,159$ & C6 & $-0,159$ \\
\hline C7 & 0,147 & C7 & 0,148 \\
\hline $\mathrm{C} 8$ & $-0,157$ & C8 & $-0,162$ \\
\hline C9 & 0,282 & C9 & 0,291 \\
\hline $\mathrm{C} 10$ & 0,041 & $\mathrm{C} 10$ & 0,042 \\
\hline C11 & $-0,245$ & C11 & $-0,245$ \\
\hline $\mathrm{C} 12$ & $-0,337$ & $\mathrm{C} 12$ & $-0,337$ \\
\hline C13 & $-0,380$ & C'1 & 0,043 \\
\hline $\mathrm{O} 14$ & $-0,552$ & $C^{\prime} 2$ & $-0,111$ \\
\hline Cl15 & $-0,024$ & C'3 & $-0,104$ \\
\hline & & $C^{\prime} 4$ & $-0,081$ \\
\hline & & C'5 & $-0,099$ \\
\hline & & C'6 & $-0,104$ \\
\hline & & $\mathrm{Cl} 13$ & $-0,024$ \\
\hline & & O14 & $-0,550$ \\
\hline
\end{tabular}

\section{Sonuç ve Öneriler}

Bu çalışmada, 7-Etil-6-klor-2-metilkromon (I) ve 7-Etil-6-klorflavon (II) sentezlendi ve kimyasal yapıları deneysel ve teorik ${ }^{1} \mathrm{H}$ NMR ve IR sonuçları kullanılarak karakterize edildi. Her iki bileşiğin deneysel ${ }^{1} \mathrm{H}$ NMR kimyasal kayma değerleri ile B3LYP/6-31G (d,p) temel seti kullanılarak hesaplanan kimyasal kayma değerleri karşılaştırıldı ve sonuçların birbiriyle uyumu incelendi. Bileşiklerin sentezinde başlangıç maddesi olarak kullanılan 4-klor-3-etilfenol'in $\mathrm{OH}$ protonlarına ait 5,30 ppm'de görülen piklerin I ve II bileşiklerinin ${ }^{1} \mathrm{H}$ NMR spektrumunda kaybolması ve kromon halkasına ait karakteristik CH piklerinin deneysel ve teorik olarak bileşik I için 6,10 ve 6,03 ppm'de, bileşik II için ise 6,80 ve 6,55 ppm'de gözlenmesi bileşiklerin kimyasal yapısını kanıtlamaktadır. Hesaplanan bă açıları ve bağ uzunlukları incelendiğinde literatür ile uyumlu olduğu görülmektedir. Her iki bileşikte beklendiği gibi en uzun bağların C-Cl bağlarına ait olduğu belirlenmiştir. IR sonuçları incelendiğinde başlangıç maddesi olarak kullanılan 4-klor-3-etilfenol'in $\mathrm{OH}$ gerilimine ait $3300-3600 \mathrm{~cm}^{-1}$ arasında ortaya çıkan pik her iki üründe de görülmemektedir. Kromon halkası için IR spektrumundaki karakteristik $\mathrm{C}=\mathrm{O}$ gerilme titreşim frekansları her iki bileşik için sırasıyla teorik ve deneysel olarak 1683 ile $1615 \mathrm{~cm}^{-1}$ ve 1657 ile $1632 \mathrm{~cm}^{-1}$ olarak görülmektedir. Sentezlenen bileşikler için HOMO ve LUMO enerji farkı $(\Delta \mathrm{E})$ sirasıyla $-4,921$ ve $-4,498 \mathrm{eV}$ olarak hesaplanmıştır ve bu yüksek enerji aralığı moleküllerin kararlı yapıda olduğunun ve kimyasal reaktivitelerinin nispeten düşük olduğunun bir göstergesidir. Sonuç olarak, 7-Etil-6-klor-2-metilkromon ve 7-Etil-6-klorflavonun sentezi ve teorik hesaplamaları üzerine literatürde herhangi bir çalışmaya rastlanmamıştır. Bileşiklerin deneysel ve teorik ${ }^{1} \mathrm{H}$ NMR ve IR sonuçları kimyasal yapılarını desteklemektedir. Ayrıca, deneysel ve teorik çalışmalardan elde edilen sonuçların birbiriyle uyumlu olduğu gözlenmiştir.

\section{Yazarların Katkısı}

Makalede tüm katkı şahsıma aittir.

\section{Çıkar Çatışması Beyanı}

Yazarlar arasında herhangi bir çıkar çatışması bulunmamaktadır.

\section{Araştırma ve Yayın Etiği Beyanı}

Yapılan çalışmada, araştırma ve yayın etiğine uyulmuştur. 


\section{Kaynaklar}

[1] Gourdeau H., Leblond L., Hamelin B., Desputeau C., Dong K., Kianicka I., Custeau D., Boudreau C., Geerts L., Cai S.X., Drewe J., Labrecque D., Kasibhatla S., Tseng B. 2014. Antivascular and antitumor evaluation of 2-amino-4-(3-bromo-4,5-dimethoxy-phenyl)-3-cyano-4H-chromenes, a novel series of anticancer agents. Molecular Cancer Therapeutics, 3 (11): 1375-1384.

[2] Sangani C.B., Shah N.M., Patel M.P., Patel R.G. 2012. Microwave-assisted synthesis of novel 4H-chromene derivatives bearing phenoxypyrazole and their antimicrobial activity assessment. Journal of the Serbian Chemical Society, 77 (9): 1165-1174.

[3] Mladenović M., Mihailović M., Bogojević D., Matić S., Nićiforović N., Mihailović V., Vuković N.,Sukdolak S.,SolujićS. 2011. In Vitro Antioxidant Activity of Selected 4-Hydroxy-chromene2-one Derivatives SAR, QSAR and DFT Studies. International Journal of Molecular Sciences, 12 (5): 2822-2841.

[4] Cheng J.F., Ishikawa A., Ono Y., Arrhenius T., Nadzan A. 2003. Novel Chromene Derivatives as TNF- $\alpha$ Inhibitors. Bioorganic\&Medicinal Chemistry Letters, 13 (21): 3647-3650.

[5] Thareja S., Verma A., Kalra A., Gosain S., Rewatkar P.V., Kokil G.R. 2010. Novel Chromeneimidazole Derivatives As Antifungal Compounds: Synthesis and In Vitro Evaluation. Acta Poloniae Pharmaceutica Drug Research, 67 (4): 423-427.

[6] Jain N., Xu J., Kanojia R.M., Du F., Jian-Zhong G., Pacia E., Lai M.T., Musto A., Allan G., Reuman M., Li X., Hahn D., Cousineau M., Peng S., Ritchie D., Russell R., Lundeen S., Sui Z. 2009. Identification and Structure-Activity Relationships of Chromene-Derived Selective Estrogen Receptor Modulators for Treatment of Postmenopausal Symptoms. Journal of Medicinal Chemistry, 52 (23): 7544-7569.

[7] Mori J., Iwashima M., Takeuchi M., Saito H. 2006. A Synthetic Study on Antiviral and Antioxidative Chromene Derivative. Chemical and Pharmaceutical Bulletin, 54 (3): 391-396.

[8] Kandeel M.M. , Aliaa M.K., Abdelall E.K.A. 2012. Design and synthesis of substituted chromenes as potential anticancer agents. International Journal of Pharmaceutical Research \& Development, 4 (3): 310-322.

[9] Karia D.C., Pandya H.K., Godvani N.K. 2012. Synthesis, Characterization \& Anti-Hiv Activity of 4-Hydroxy-3-(5-Methylisoxazol-3-Yl) Pyrano (3,2-C) Chromene-2,5-Dione. Asian Journal of Biochemical and Pharmaceutical Research, 2 (2): 126-130.

[10] Kamdar N.R., Haveliwala D.D., Mistry P.T., Patel S. 2011. Synthesis and evaluation of in vitro antitubercular activity and antimicrobial activity of some novel $4 \mathrm{H}$-chromeno[2,3-d]pyrimidine via 2-amino-4-phenyl-4H-chromene-3-carbonitriles. Medicinal Chemistry Research, 20 (7): 854864.

[11] Gupta S., Kumar N., Kumar S., Dudhe R., Gupta H. 2012. 3-Hydroxy-2-(substituted phenyl) 4H-chromen-4-one derivatives- synthesis, spectral characterization and pharmacological screening. International Journal of Therapeutic Applications, 7: 1-8.

[12] Bhat M.A., Siddiqui N., Khan S.A. 2008. Synthesis of novel 3-(4-acetyl-5H/methyl-5-substituted phenyl-4,5-dihydro-1,3,4-oxadiazol-2-yl)-2H-chromen-2-ones as potential anticonvulsant agents. Acta Poloniae Pharmaceutica, 65 (2): 235-239.

[13] Thompson L.A., Ellman J.A. 1996. Synthesis and Applications of Small Molecule Libraries. Chemical Reviews, 96: 555-600.

[14] Lingen H.L., Zhuang Wei., Hansen T., Rutjes F.P.J.T., Jørgensen K.A. 2003. Formation of optically active chromanes by catalytic asymmetric tandem oxa-Michael addition-Friedel-Crafts alkylation reactions. Organic and Biomolecular Chemistry, 11: 1953-1958.

[15] Yao N., Song A., Wang X., Dixon S., Lam K.S. 2007. Synthesis of Flavonoid Analogues as Scaffolds for Natural Product-Based Combinatorial Libraries. American Chemical Society, 9: 668-676.

[16] Atohoun Y.G.S., Doco R.C. , Houngue M.T.A.K., Kuevi A.U., Kpotin G.A., Mensah J.B. 2016. Theoretical Study of antioxidant properties of three isomers flavonoids: kaempferol, luteolin and fisetin. American Journal of Scientific And Industrial Research, 7 (6): 145-152.

[17] Frisch M.J., Trucks H.B., Schlegel G.E., Scuseria M., Robb J.R., Cheeseman G., Scalmani V., Barone B., Mennucci G.A., Petersson H., Nakatsuji M., Caricato X., Li H.P., Hratchian A.F., Izmaylov J., Bloino G., Zheng J.L, Sonnenberg M., Hada M., Ehara K, Toyota R, Fukuda J, 
Hasegawa M., Ishida T., Nakajima Y., Honda O., Kitao H., Nakai T., Vreven A., Montgomery J., Peralta F., Ogliaro M., Bearpark J.J., Heyd E., Brothers K.N., Kudin V.N., Staroverov R., Kobayashi J., Normand K., Raghavachari A., Rendell J.C., Burant S.S., Iyengar J., Tomasi M., Cossi N., Rega J.M., Millam M. 2009. Gaussian Inc. Wallingford CT.

[18] Lee C., Yang W., Parr R.G. 1988. Development of the Colle- Salvetti correlation energy formula into a functional of the electron density. Physical Review B, 37 (2): 785-789.

[19] Wolinski K., Hinton J.F., Pulay P. 1990. Efficient Implementation of the Gauge-Independent Atomic Orbital Method for NMR Chemical Shift Calculations. Journal of the American Chemical Society, 112: 8251-8260.

[20] Erdoğdu Y., Ünsalan O., Güllüoğlu M.T. 2009. Vibrational analysis of flavone. Turkish Journal of Physics, 33: 249-259.

[21] Günay N., Gümüş H. Atalay Y. 2011. L-Asparaginyum Pikrat Molekülünün Spektroskopik Özelliklerinin Teorik Olarak İncelenmesi. Sakarya Üniversitesi Fen Edebiyat Dergisi, 15-32.

[22] Boopathi M. M., Udhayakala P., Ramkumaar G.R. 2016. Vibrational spectroscopic (FT-IR, FTRaman), NMR and electronic structure calculations of metaxalone. Der Pharma Chemica, 8 (7): $161-172$. 